\title{
Sublinear Operators with Rough Kernel on Herz Spaces with Variable Exponents
}

\author{
Yu Shu ${ }^{1}$, Liwei Wang ${ }^{2, *}$ and Dan $\mathrm{Xiao}^{3}$ \\ 1 School of Electronic Commerce, Anhui Business College, Wuhu 241002, Anhui, \\ China \\ 2 School of Mathematics-Physics and Finance, Anhui Polytechnic University, \\ Wuhu 241000, Anhui, China \\ ${ }^{3}$ Department of Basic Courses, Anhui Professional College of Art, Hefei 230001, \\ Anhui, China
}

Received 5 April 2020; Accepted (in revised version) 8 September 2020

\begin{abstract}
We prove some boundedness results for a large class of sublinear operators with rough kernel on the homogeneous Herz spaces where the three main indices are variable exponents. Some known results are extended.
\end{abstract}

Key Words: Herz space, variable exponent, sublinear operator.

AMS Subject Classifications: 42B35, 46E30

\section{Introduction}

Suppose that $\mathbb{S}^{n-1}$ is the unit sphere of $\mathbb{R}^{n}(n \geq 2)$ equipped with normalized Lebesgue measure $d \sigma\left(x^{\prime}\right)$. Let $\Omega \in L^{1}\left(S^{n-1}\right)$ be homogeneous of degree zero and satisfy

$$
\int_{\mathbb{S}^{n-1}} \Omega\left(x^{\prime}\right) d \sigma\left(x^{\prime}\right)=0,
$$

where $x^{\prime}=x /|x|$ for any $x \neq 0$. In this paper, we will consider sublinear operators which satisfy that for any $f \in L^{1}\left(\mathbb{R}^{n}\right)$ with compact support and $x \notin \operatorname{supp} f$,

$$
\left|T_{\Omega} f(x)\right| \leq C \int_{\mathbb{R}^{n}} \frac{|\Omega(x-y)|}{|x-y|^{n}}|f(y)| d y,
$$

and their corresponding fractional versions

$$
\left|T_{\Omega, \beta} f(x)\right| \leq C \int_{\mathbb{R}^{n}} \frac{|\Omega(x-y)|}{|x-y|^{n-\beta}}|f(y)| d y,
$$

*Corresponding author. Email addresses: shulsh@mail.ahnu.edu.cn (Y. Shu), wangliwei@ahpu.edu.cn (L. W. Wang), smilexd163@163.com (D. Xiao) 
where $0<\beta<n$ and $C>0$ is an absolute constant.

Soria and Weiss [20] first introduced the condition (1.1), which is satisfied by many classical operators in harmonic analysis, such as the Calderón-Zygmund operators, Carleson's maximal operators, Hardy-Littlewood maximal operators, etc. In the case $\Omega \in$ $L^{s}\left(\mathbb{S}^{n-1}\right)$ for some $s \in[1, \infty]$, Lu et al. [15] proved the boundedness of sublinear operators $T_{\Omega}$ and $T_{\Omega, \beta}$ on generalized Morrey spaces. Hu et al. [10] established the boundedness of sublinear operators with rough kernel on the classical Herz spaces. We refer to [13] for further results on these operators.

In recent years, function spaces with variable exponents have attracted more and more attention. The growing interest in such spaces is strongly stimulated by the treatment of recent problems in fluid dynamics [19], image restoration [3] and PDE with non-standard growth conditions [7]. The generalized Lebesgue spaces $L^{p(\cdot)}\left(\mathbb{R}^{n}\right)$ (also known as Lebesgue spaces with variable exponent) and the corresponding generalized Sobolev spaces $W^{k, p(\cdot)}\left(\mathbb{R}^{n}\right)$ have been systematically studied by Kováčik and Rákosník in [12]. Since then various other function spaces such as Herz spaces [11], Morrey type spaces $[8,16]$ and so on have been investigated in the variable exponent setting.

As shown in $[14,18]$, Herz spaces play a crucial role in harmonic analysis and PDE. For instance, they appear in the characterization of multiplier on Hardy spaces and in the regularity theory for elliptic and parabolic equations in divergence form. Herz spaces $\dot{K}_{p(\cdot), q}^{\alpha(\cdot)}\left(\mathbb{R}^{n}\right)$ and $K_{p(\cdot), q}^{\alpha(\cdot)}\left(\mathbb{R}^{n}\right)$ with variable exponent $\alpha, p$ but fixed $q \in \mathbb{R}$ were first studied by Almeida and Drihem [1], and they also studied the boundedness of a wide class of sublinear operators on these spaces. Recently, Drihem and Seghiri in [6] generalized some of the main results in [1] to the Herz spaces $\dot{K}_{p(\cdot), q(\cdot)}^{\alpha(\cdot)}\left(\mathbb{R}^{n}\right)$ and $K_{p(\cdot), q(\cdot)}^{\alpha(\cdot)}\left(\mathbb{R}^{n}\right)$, where the exponent $q$ is variable as well. The main purpose of this paper is to further extend these results to the rough kernel case.

In general, by $B$ we denote the ball with center $x \in \mathbb{R}^{n}$ and radius $r>0$. If $E$ is a subset of $\mathbb{R}^{n},|E|$ denotes its Lebesgue measure and $\chi_{E}$ its characteristic function. $p^{\prime}$ denotes the conjugate exponent defined by $\frac{1}{p}+\frac{1}{p^{\prime}}=1$. We use $x \approx y$ if there exist constants $c_{1}, c_{2}$ such that $c_{1} x \leq y \leq c_{2} x$. The symbol $C$ stands for a positive constant, which may vary from line to line.

\section{Preliminaries and lemmas}

We begin with a brief and necessarily incomplete review of the variable exponent Lebesgue spaces $L^{p(\cdot)}\left(\mathbb{R}^{n}\right)$, see $[4,5]$ for more information.

Let $\mathcal{P}\left(\mathbb{R}^{n}\right)$ denote the set of all measurable functions $p(\cdot): \mathbb{R}^{n} \rightarrow[1, \infty)$. For $p(\cdot) \in$ $\mathcal{P}\left(\mathbb{R}^{n}\right)$, we use the notation

$$
p_{-}:=\operatorname{ess} \inf _{x \in \mathbb{R}^{n}} p(x), \quad p_{+}:=\text {ess } \sup _{x \in \mathbb{R}^{n}} p(x)
$$


The Lebesgue space with variable exponent $L^{p(\cdot)}\left(\mathbb{R}^{n}\right)$ is the class of all measurable functions $f$ on $\mathbb{R}^{n}$ such that

$$
I_{p(\cdot)}(f):=\int_{\mathbb{R}^{n}}|f(x)|^{p(x)} d x<\infty .
$$

This is a Banach space with respect to the Luxemburg norm

$$
\|f\|_{L^{p(\cdot)}\left(\mathbb{R}^{n}\right)}=\inf \left\{\lambda>0: I_{p(\cdot)}(f / \lambda) \leq 1\right\} .
$$

It is obvious that the variable exponent Lebesgue norm has the following property

$$
\left\||f|^{\sigma}\right\|_{L^{p(\cdot)}\left(\mathbb{R}^{n}\right)}=\|f\|_{L^{\sigma p(\cdot)}\left(\mathbb{R}^{n}\right)^{\prime}}^{\sigma} \quad \sigma \geq 1 / p_{-} .
$$

Given an open set $\Omega \subset \mathbb{R}^{n}$, the space $L_{\text {loc }}^{p(\cdot)}(\Omega)$ is defined by

$$
L_{\text {loc }}^{p(\cdot)}(\Omega)=\left\{f: f \in L^{p(\cdot)}(F) \text { for all compact subsets } F \subset \Omega\right\} .
$$

For $p(\cdot) \in \mathcal{P}\left(\mathbb{R}^{n}\right)$, Hölder's inequality (see [12, Theorem 2.1]) holds in the form

$$
\int_{\mathbb{R}^{n}}|f(x) g(x)| d x \leq\left(1+\frac{1}{p_{-}}-\frac{1}{p_{+}}\right)\|f\|_{L^{p(\cdot)}\left(\mathbb{R}^{n}\right)}\|g\|_{L^{p^{\prime}(\cdot)}\left(\mathbb{R}^{n}\right)^{\prime}}
$$

where and in the sequel $p^{\prime}(x)=\frac{p(x)}{p(x)-1}$ is the conjugate function of $p(x)$.

For our main results we need to impose some regularities on the exponent function $p(\cdot)$. The most important condition, one widely used in the study of variable Lebesgue spaces, is so-called log-Hölder continuity. Given a function $\phi(\cdot): \mathbb{R}^{n} \rightarrow \mathbb{R}$, we say $\phi(\cdot)$ is locally $\log$-Hölder continuous if there exists a constant $C_{\log }>0$ such that

$$
|\phi(x)-\phi(y)| \leq \frac{C_{\log }}{\log (e+1 /|x-y|)}, \quad \forall x, y \in \mathbb{R}^{n} .
$$

If, for some $\phi_{\infty} \in \mathbb{R}$ and $C_{\log }>0$, there holds

$$
\begin{array}{ll}
|\phi(x)-\phi(0)| \leq \frac{C_{\log }}{\log (e+1 /|x|)}, & \forall x \in \mathbb{R}^{n}, \\
\left|\phi(x)-\phi_{\infty}\right| \leq \frac{C_{\log }}{\log (e+|x|)}, & \forall x \in \mathbb{R}^{n},
\end{array}
$$

then we say $\phi(\cdot)$ is log-Hölder continuous at the origin (or has a log decay at the origin) and at infinity (or has a log decay at infinity), respectively.

By $\mathcal{P}_{0}^{\log }\left(\mathbb{R}^{n}\right)$ and $\mathcal{P}_{\infty}^{\log }\left(\mathbb{R}^{n}\right)$ we denote the class of exponents $p(\cdot) \in \mathcal{P}\left(\mathbb{R}^{n}\right)$, which satisfy conditions (2.4a) and (2.4b), respectively. $\mathcal{P}^{\log }\left(\mathbb{R}^{n}\right)$ is the set of functions $p(\cdot) \in$ $\mathcal{P}\left(\mathbb{R}^{n}\right)$ satisfying conditions (2.3) and (2.4b), with $p_{\infty}:=\lim _{|x| \rightarrow \infty} p(x)$. It is easy to see that $\mathcal{P}^{\log }\left(\mathbb{R}^{n}\right) \subset \mathcal{P}_{0}^{\log }\left(\mathbb{R}^{n}\right) \cap \mathcal{P}_{\infty}^{\log }\left(\mathbb{R}^{n}\right)$ and $p(\cdot) \in \mathcal{P}^{\log }\left(\mathbb{R}^{n}\right)$ if and only if $p^{\prime}(\cdot) \in \mathcal{P}^{\log }\left(\mathbb{R}^{n}\right)$. 
In particular, we note that if $p(\cdot) \in \mathcal{P}^{\log }\left(\mathbb{R}^{n}\right)$ with $1<p_{-} \leq p_{+}<\infty$, then the HardyLittlewood maximal operator $M$ defined by

$$
M f(x)=\sup _{x \in \mathbb{R}^{n}, r>0} \frac{1}{|B(x, r)|} \int_{B(x, r)}|f(y)| d y,
$$

is bounded on $L^{p(\cdot)}\left(\mathbb{R}^{n}\right)$, see [5, Theorem 4.3.8].

By $p^{*}(\cdot)$ we denote the Sobolev exponent defined by $1 / p^{*}(x):=1 / p(x)-\beta / n, 0<$ $\beta<n$. We note that if $p(\cdot)$ is locally log-Hölder continuous and has a log decay at infinity, $1 \leq p_{-} \leq p_{+}<\infty$ and $0<\beta<n / p_{+}$, then $p^{*}(\cdot)$ is locally log-Hölder continuous and has a log decay at infinity, and

$$
1<\frac{n p_{-}}{n-\beta p_{-}}=\left(p^{*}\right)_{-} \leq\left(p^{*}\right)_{+}=\frac{n p_{+}}{n-\beta p_{+}}<\infty .
$$

Moreover, we can show that the assumption $p(\cdot) \in \mathcal{P} \log \left(\mathbb{R}^{n}\right)$ implies $p^{*}(\cdot) \in \mathcal{P} \log \left(\mathbb{R}^{n}\right)$, see $[1,9]$ for further details.

Next, let us introduce some lemmas. We remark that Lemma 2.1 is due to Nakai and Sawano [17, p. 3681]. Lemmas 2.2-2.4 were shown in Almeida and Drihem [1].

Lemma 2.1. Let $p(\cdot) \in \mathcal{P}\left(\mathbb{R}^{n}\right)$. If $q>p_{+}$and $\frac{1}{p(x)}=\frac{1}{\tilde{q}(x)}+\frac{1}{q}$, then we have

$$
\|f g\|_{L^{p(\cdot)}\left(\mathbb{R}^{n}\right)} \leq C\|f\|_{L^{\tilde{q}(\cdot)}\left(\mathbb{R}^{n}\right)}\|g\|_{L^{q}\left(\mathbb{R}^{n}\right)}
$$

for all measurable functions $f$ and $g$.

Lemma 2.2. Let $r_{1}>0$. Suppose $\alpha(\cdot) \in L^{\infty}\left(\mathbb{R}^{n}\right)$ is log-Hölder continuous both at the origin and at infinity, then we have

$$
r_{1}^{\alpha(x)} \leq C r_{2}^{\alpha(y)} \times \begin{cases}\left(\frac{r_{1}}{r_{2}}\right)^{\alpha_{+}}, & 0<r_{2} \leq r_{1} / 2, \\ 1, & r_{1} / 2<r_{2} \leq 2 r_{1}, \\ \left(\frac{r_{1}}{r_{2}}\right)^{\alpha_{-}}, & r_{2}>2 r_{1},\end{cases}
$$

for any $x \in B\left(0, r_{1}\right) \backslash B\left(0, r_{1} / 2\right)$ and $y \in B\left(0, r_{2}\right) \backslash B\left(0, r_{2} / 2\right)$.

Lemma 2.3. Let $p(\cdot) \in \mathcal{P}_{\infty}^{\log }\left(\mathbb{R}^{n}\right)$ and $R=B(0, r) \backslash B(0, r / 2)$. If $|R| \geq 2^{-n}$, then

$$
\left\|\chi_{R}\right\|_{L^{p(\cdot)}\left(\mathbb{R}^{n}\right)} \approx|R|^{\frac{1}{p(x)}} \approx|R|^{\frac{1}{p_{\infty}}} .
$$

The left-hand side equivalence remains true for every $|R|>0$ if we assume, additionally, $p(\cdot) \in$ $\mathcal{P}_{0}^{\log }\left(\mathbb{R}^{n}\right) \cap \mathcal{P}_{\infty}^{\log }\left(\mathbb{R}^{n}\right)$.

By $\ell^{q}$, we denote the discrete Lebesgue space equipped with the usual quasinorm. As a consequence of Young's inequality in the sequence Lebesgue space $\ell^{q}$, we have the following statement. 
Lemma 2.4. Let $0<a<1$ and $0<q \leq \infty$. Let $\left\{\varepsilon_{k}\right\}_{k \in \mathbb{Z}}$ be a sequence of positive real numbers, such that

$$
\left\|\left\{\varepsilon_{k}\right\}_{k \in \mathbb{Z}}\right\|_{\ell q}=I<\infty .
$$

Then the sequences

$$
\left\{\xi_{k}: \xi_{k}=\sum_{j \leq k} a^{k-j} \varepsilon_{j}\right\}_{k \in \mathbb{Z}} \quad \text { and } \quad\left\{\eta_{k}: \eta_{k}=\sum_{j \geq k} a^{j-k} \varepsilon_{j}\right\}_{k \in \mathbb{Z}}
$$

belong to $\ell^{q}$, and

$$
\left\|\left\{\xi_{k}\right\}_{k \in \mathbb{Z}}\right\|_{\ell^{q}}+\left\|\left\{\eta_{k}\right\}_{k \in \mathbb{Z}}\right\|_{\ell^{q}} \leq C I,
$$

with the implicit constant only depending on $a$ and $q$.

\section{Main results and their proofs}

In what follows, by $\mathcal{P}_{0}\left(\mathbb{R}^{n}\right)$ we denote the set of measurable functions on $\mathbb{R}^{n}$ with range in $[c,+\infty)$ for some $c>0$. By $\mathcal{P}_{+}\left(\mathbb{R}^{n}\right)$ we denote the set of variable exponents $p(\cdot) \in \mathcal{P}\left(\mathbb{R}^{n}\right)$ with $1<p_{-} \leq p_{+}<\infty$.

Let $p(\cdot), q(\cdot) \in \mathcal{P}_{0}\left(\mathbb{R}^{n}\right)$. Define the mixed Lebesgue sequence space $\ell^{q(\cdot)}\left(L^{p(\cdot)}\right)$ to be the set of all sequences $\left\{f_{k}\right\}_{k=0}^{\infty}$ of measurable functions on $\mathbb{R}^{n}$ such that

$$
\left\|\left\{f_{k}\right\}_{k=0}^{\infty}\right\|_{\ell^{q(\cdot)}\left(L^{p(\cdot)}\right)}:=\inf \left\{\mu>0: \rho_{\ell^{q(\cdot)}\left(L^{p(\cdot)}\right)}\left(\left\{\frac{f_{k}}{\mu}\right\}_{k=0}^{\infty}\right) \leq 1\right\}<\infty,
$$

where

$$
\rho_{\ell^{q}(\cdot)\left(L^{p(\cdot)}\right)}\left(\left\{f_{k}\right\}_{k=0}^{\infty}\right):=\sum_{k \geq 0} \inf \left\{\lambda_{k}: \int_{\mathbb{R}^{n}}\left(\frac{\left|f_{k}(x)\right|}{\lambda_{k}^{\frac{1}{q(x)}}}\right)^{p(x)} d x \leq 1\right\} .
$$

Since $q_{+}<\infty$, then we have

$$
\rho_{\ell q(\cdot)\left(L^{p(\cdot)}\right)}\left(\left\{f_{k}\right\}_{k=0}^{\infty}\right)=\sum_{k \geq 0}\left\|\left|f_{k}\right|^{q(\cdot)}\right\|_{L^{\frac{p(\cdot)}{q(\cdot)}}} .
$$

Furthermore, if $p$ and $q$ are constants, then $\ell^{q(\cdot)}\left(L^{p(\cdot)}\right)=\ell^{q}\left(L^{p}\right)$. It is known that $\|$. $\|_{\ell^{q(\cdot)}\left(L^{p(\cdot)}\right)}$ is a quasi-norm for all $p(\cdot), q(\cdot) \in \mathcal{P}_{0}\left(\mathbb{R}^{n}\right)$ and that $\|\cdot\|_{\ell^{q(\cdot)}\left(L^{p(\cdot)}\right)}$ is a norm when $\frac{1}{p(\cdot)}+\frac{1}{q(\cdot)} \leq 1$, see [2] for further details.

Here and below, we set

$$
B_{k}:=\left\{x \in \mathbb{R}^{n}:|x| \leq 2^{k}\right\}, \quad R_{k}:=B_{k} \backslash B_{k-1} \quad \text { and } \quad \chi_{k}=\chi_{R_{k}}, \quad k \in \mathbb{Z} .
$$

Definition 3.1. Let $p(\cdot), q(\cdot) \in \mathcal{P}_{0}\left(\mathbb{R}^{n}\right)$ and $\alpha(\cdot): \mathbb{R}^{n} \rightarrow \mathbb{R}$ with $\alpha(\cdot) \in L^{\infty}\left(\mathbb{R}^{n}\right)$. The homogeneous Herz space $\dot{K}_{p(\cdot), q(\cdot)}^{\alpha(\cdot)}\left(\mathbb{R}^{n}\right)$ is defined as the set of all $f \in L_{\mathrm{loc}}^{p(\cdot)}\left(\mathbb{R}^{n} \backslash\{0\}\right)$ such that

$$
\|f\|_{\dot{K}_{p(\cdot), q(\cdot)}^{\alpha(\cdot)}\left(\mathbb{R}^{n)}\right.}:=\left\|\left(2^{k \alpha(\cdot)} f \chi_{k}\right)_{k \in \mathbb{Z}}\right\|_{\ell^{q(\cdot)}\left(L^{p(\cdot)}\right)}<\infty .
$$


Obviously, Herz spaces $\dot{K}_{p(\cdot), q(\cdot)}^{\alpha(\cdot)}\left(\mathbb{R}^{n}\right)$ can be regarded as a generalization of $\dot{K}_{p(\cdot)}^{\alpha, q}\left(\mathbb{R}^{n}\right)$ and $\dot{K}_{p(\cdot), q}^{\alpha(\cdot)}\left(\mathbb{R}^{n}\right)$ defined in [11] and [1], respectively. If both $\alpha(\cdot), p(\cdot)$ and $q(\cdot)$ are constants, then $\dot{K}_{p(\cdot), q(\cdot)}^{\alpha(\cdot)}\left(\mathbb{R}^{n}\right)$ coincides with the classical Herz spaces.

Let us denote

$$
\left\|\left\{h_{k}\right\}\right\|_{\ell_{>}^{q}\left(L^{p(\cdot)}\right)}=\left(\sum_{k \geq 0}\left\|h_{k}\right\|_{L^{p(\cdot)}\left(\mathbb{R}^{n}\right)}^{q}\right)^{1 / q} \text { and }\left\|\left\{h_{k}\right\}\right\|_{\ell_{<}^{q}\left(L^{p(\cdot)}\right)}=\left(\sum_{k<0}\left\|h_{k}\right\|_{L^{p(\cdot)}\left(\mathbb{R}^{n}\right)}^{q}\right)^{1 / q}
$$

for sequences $\left\{h_{k}\right\}_{k \in \mathbb{Z}}$ of measurable functions (with the usual modification when $q=$ $\infty)$

Drihem and Seghiri in [6] obtained the following result.

Proposition 3.1. Let $p(\cdot), q(\cdot) \in \mathcal{P}_{0}\left(\mathbb{R}^{n}\right)$ and $\alpha(\cdot) \in L^{\infty}\left(\mathbb{R}^{n}\right)$. If both $\alpha(\cdot), q(\cdot)$ are log-Hölder continuous at the origin and at infinity, then

$$
\|f\|_{\dot{K}_{p(\cdot), q(\cdot)}^{\alpha(\cdot)}\left(\mathbb{R}^{n}\right)} \approx\left\|\left\{2^{\alpha(0) k} f \chi_{k}\right\}\right\|_{\ell_{<}^{q(0)}\left(L^{p(\cdot)}\right)}+\left\|\left\{2^{\alpha_{\infty} k} f \chi_{k}\right\}\right\|_{\ell_{>}^{q_{\infty}}\left(L^{p(\cdot)}\right)} .
$$

The main results obtained in this paper are as follows.

Theorem 3.1. Suppose $q(\cdot) \in \mathcal{P}_{0}\left(\mathbb{R}^{n}\right), p(\cdot) \in \mathcal{P}_{+}\left(\mathbb{R}^{n}\right) \cap \mathcal{P}_{0}^{\log }\left(\mathbb{R}^{n}\right) \cap \mathcal{P}_{\infty}^{\log }\left(\mathbb{R}^{n}\right)$ and $\Omega \in$ $L^{s}\left(S^{n-1}\right)$ with $\left(p^{\prime}\right)_{+}<s \leq \infty$. Let $\alpha(\cdot) \in L^{\infty}\left(\mathbb{R}^{n}\right)$ be log-Hölder continuous both at the origin and at infinity, such that

$$
-\frac{n}{p_{+}}<\alpha_{-} \leq \alpha_{+}<n\left(1-\frac{1}{p_{-}}\right)-\frac{n-1}{s}
$$

Then every sublinear operator $T_{\Omega}$ satisfying condition (1.1) which is bounded on $L^{p(\cdot)}\left(\mathbb{R}^{n}\right)$ is also bounded on $\dot{K}_{p(\cdot), q(\cdot)}^{\alpha(\cdot)}\left(\mathbb{R}^{n}\right)$.

Remark 3.1. In the case $\Omega$ is constant, the corresponding statement to Theorem 3.1 was proved by Almeida and Drihem [1], with variable exponents $\alpha, p$ but fixed $q \in(0, \infty]$. Theorem 3.1 is also a generalization of Drihem and Seghiri's result in [6, Theorem 2].

Theorem 3.2. Suppose $0<\beta<n, q(\cdot) \in \mathcal{P}_{0}\left(\mathbb{R}^{n}\right), p(\cdot) \in \mathcal{P}_{0}^{\log }\left(\mathbb{R}^{n}\right) \cap \mathcal{P}_{\infty}^{\log }\left(\mathbb{R}^{n}\right)$ with $1<$ $p_{-} \leq p_{+}<n / \beta$ and $\Omega \in L^{s}\left(S^{n-1}\right),\left(p^{\prime}\right)_{+}<s \leq \infty$. Let $\alpha(\cdot) \in L^{\infty}\left(\mathbb{R}^{n}\right)$ be log-Hölder continuous both at the origin and at infinity, such that

$$
\beta-\frac{n}{p_{+}}<\alpha_{-} \leq \alpha_{+}<n\left(1-\frac{1}{p_{-}}\right)-\frac{n-1}{s} .
$$

Then every sublinear operator $T_{\Omega, \beta}$ satisfying condition (1.2) which is bounded from $L^{p(\cdot)}\left(\mathbb{R}^{n}\right)$ into $L^{p^{*}(\cdot)}\left(\mathbb{R}^{n}\right)$ is also bounded from $\dot{K}_{p(\cdot), q(\cdot)}^{\alpha(\cdot)}\left(\mathbb{R}^{n}\right)$ into $\dot{K}_{p^{*}(\cdot), q(\cdot)}^{\alpha(\cdot)}\left(\mathbb{R}^{n}\right)$. 
Obviously, if $\Omega$ is constant, then the Riesz potential operator

$$
I_{\beta} f(x):=\int_{\mathbb{R}^{n}} \frac{f(y)}{|x-y|^{n-\beta}} d y
$$

and the fractional maximal function

$$
M_{\beta} f(x):=\sup _{x \in \mathbb{R}^{n}, r>0} \frac{1}{|B(x, r)|^{1-\frac{\beta}{n}}} \int_{B(x, r)}|f(y)| d y
$$

both satisfy the size condition (1.2). In view of the well-known pointwise estimate $M_{\beta} f(x) \leq C I_{\beta}(|f|)(x)$ and the $\left(L^{p(\cdot)}, L^{p^{*}(\cdot)}\right)$-boundedness of $I_{\beta}$ for $p(\cdot) \in \mathcal{P} \log \left(\mathbb{R}^{n}\right)$ with $1<p_{-} \leq p_{+}<n / \beta$ (see [5, Theorem 6.1.9]), from Theorem 3.2, we get the following.

Corollary 3.1. Suppose $0<\beta<n, q(\cdot) \in \mathcal{P}_{0}\left(\mathbb{R}^{n}\right)$ and $p(\cdot) \in \mathcal{P}^{\log }\left(\mathbb{R}^{n}\right)$ with $1<p_{-} \leq$ $p_{+}<n / \beta$. Let $\alpha(\cdot) \in L^{\infty}\left(\mathbb{R}^{n}\right)$ be log-Hölder continuous both at the origin and at infinity, such that

$$
\beta-\frac{n}{p_{+}}<\alpha_{-} \leq \alpha_{+}<n\left(1-\frac{1}{p_{-}}\right) .
$$

Then $I_{\beta}$ and $M_{\beta}$ are bounded from $\dot{K}_{p(\cdot), q(\cdot)}^{\alpha(\cdot)}\left(\mathbb{R}^{n}\right)$ into $\dot{K}_{p^{*}(\cdot), q(\cdot)}^{\alpha(\cdot)}\left(\mathbb{R}^{n}\right)$.

In fact, without essential difficulties, one can prove Theorem 3.1 by using the similar arguments as in the proof of Theorem 3.2. Thus we need only to prove Theorem 3.2.

Proof of Theorem 3.2. In view of Proposition 3.1, we split the operator into

$$
\left|T_{\Omega, \beta} f(x)\right| \leq\left|T_{\Omega, \beta}\left(f \chi_{B_{k-2}}\right)(x)\right|+\left|T_{\Omega, \beta}\left(f \chi_{\widetilde{R_{k}}}\right)(x)\right|+\left|T_{\Omega, \beta}\left(f \chi_{\mathbb{R}^{n} \backslash B_{k+2}}\right)(x)\right|,
$$

where $\widetilde{R_{k}}:=\left\{x \in \mathbb{R}^{n}: 2^{k-2} \leq|x|<2^{k+2}\right\}$ with $k \in \mathbb{Z}$ and $x \in R_{k}$.

To estimate $T_{\Omega, \beta}\left(f \chi_{B_{k-2}}\right)$, we write

$$
\begin{aligned}
& 2^{k \alpha(0)}\left|T_{\Omega, \beta}\left(f \chi_{B_{k-2}}\right)(x)\right| \\
\leq & C 2^{k \alpha(0)} \int_{B_{k-2}} \frac{|\Omega(x-y)|}{|x-y|^{n-\beta}}|f(y)| d y \\
= & C 2^{k \alpha(0)} \sum_{j=-\infty}^{k-2} \int_{R_{j}} \frac{|\Omega(x-y)|}{|x-y|^{n-\beta}}|f(y)| d y .
\end{aligned}
$$

Note that if $x \in R_{k}, k<0$, then

$$
|x-y| \geq|x|-|y|>\frac{2^{k}}{4} \quad \text { and } \quad 2^{k \alpha(x)} \approx 2^{k \alpha(0)} .
$$

Hence by Lemma 2.2, we arrive at the inequality

$$
2^{k \alpha(0)}\left|T_{\Omega, \beta}\left(f \chi_{B_{k-2}}\right)(x)\right| \leq C \sum_{j=-\infty}^{k-2} 2^{(k-j) \alpha_{+}-k(n-\beta)} \int_{R_{j}} 2^{j \alpha(y)}|\Omega(x-y)||f(y)| d y .
$$


This together with Hölder's inequality (2.2) gives

$$
\begin{aligned}
& \left\|2^{k \alpha(0)} T_{\Omega, \beta}\left(f \chi_{B_{k-2}}\right) \chi_{k}\right\|_{L^{p^{*}(\cdot)}\left(\mathbb{R}^{n}\right)} \\
\leq C & \sum_{j=-\infty}^{k-2} 2^{(k-j) \alpha_{+}-k(n-\beta)}\left\|2^{j \alpha(\cdot)} f \chi_{j}\right\|_{L^{p(\cdot)}\left(\mathbb{R}^{n}\right)}\left\|\Omega(x-\cdot) \chi_{j}\right\|_{L^{p^{(\cdot)}\left(\mathbb{R}^{n}\right)}}\left\|\chi_{k}\right\|_{L^{p^{*}(\cdot)}\left(\mathbb{R}^{n}\right)} .
\end{aligned}
$$

Since $s>\left(p^{\prime}\right)_{+}$, we can define a variable exponent $\widetilde{p}(\cdot)$ by

$$
\frac{1}{p^{\prime}(x)}=\frac{1}{\widetilde{p}(x)}+\frac{1}{s^{\prime}}
$$

then by Lemma 2.1 and Lemma 2.3, we have

$$
\begin{aligned}
& \left\|\Omega(x-\cdot) \chi_{j}\right\|_{L^{p^{\prime}(\cdot)\left(\mathbb{R}^{n}\right)}} \\
\leq & C\left\|\Omega(x-\cdot) \chi_{j}\right\|_{L^{s}\left(\mathbb{R}^{n}\right)}\left\|\chi_{j}\right\|_{L^{\tilde{p}(\cdot)}\left(\mathbb{R}^{n}\right)} \\
\leq & C\left\|\chi_{j}\right\|_{L^{p^{\prime}(\cdot)}\left(\mathbb{R}^{n}\right)}\left|B_{j}\right|^{-1 / s}\left(\int_{|x|-2^{j}}^{|x|+2^{j}} \int_{S^{n-1}}\left|\Omega\left(y^{\prime}\right)\right|^{s} d \sigma\left(y^{\prime}\right) \rho^{n-1} d \rho\right)^{\frac{1}{s}} \\
\leq & C 2^{(k-j)(n-1) / s}\left\|\chi_{j}\right\|_{L^{p^{\prime}(\cdot)\left(\mathbb{R}^{n}\right)}} .
\end{aligned}
$$

Observe that

$$
\left|R_{j}\right|^{-\frac{1}{p\left(x_{j}\right)}}\left|R_{k}\right|^{\frac{1}{p\left(x_{k}\right)}} \leq C 2^{(k-j) \frac{n}{p_{-}}}
$$

for $x_{j} \in R_{j}, x_{k} \in R_{k}$ and $j \leq k-2$ (see [1, p. 790]). Since $p(\cdot) \in \mathcal{P}_{0}^{\log }\left(\mathbb{R}^{n}\right) \cap \mathcal{P}_{\infty}^{\log }\left(\mathbb{R}^{n}\right)$ implies that $p^{\prime}(\cdot), p^{*}(\cdot) \in \mathcal{P}_{0}^{\log }\left(\mathbb{R}^{n}\right) \cap \mathcal{P}_{\infty}^{\log }\left(\mathbb{R}^{n}\right)$, from (3.2), (3.3) and Lemma 2.3, it follows that

$$
\begin{aligned}
& \left\|2^{k \alpha(0)} T_{\Omega, \beta}\left(f \chi_{B_{k-2}}\right) \chi_{k}\right\|_{L^{p^{*}(\cdot)}\left(\mathbb{R}^{n}\right)} \\
\leq & C \sum_{j=-\infty}^{k-2} 2^{(k-j) \alpha_{+}-k(n-\beta)} 2^{(k-j)(n-1) / s}\left\|2^{j \alpha(\cdot)} f \chi_{j}\right\|_{L^{p(\cdot)}\left(\mathbb{R}^{n}\right)}\left\|\chi_{j}\right\|_{L^{p^{(\cdot)}\left(\mathbb{R}^{n}\right)}}\left\|\chi_{k}\right\|_{L^{p^{*}(\cdot)}\left(\mathbb{R}^{n}\right)} \\
\leq & C \sum_{j=-\infty}^{k-2} 2^{(k-j)\left(\alpha_{+}-n\right)} 2^{(k-j)(n-1) / s}\left\|2^{j \alpha(\cdot)} f \chi_{j}\right\|_{L^{p(\cdot)}\left(\mathbb{R}^{n}\right)}\left|R_{j}\right|^{-\frac{1}{p\left(x_{j}\right)}}\left|R_{k}\right|^{\frac{1}{p\left(x_{k}\right)}} \\
\leq & C \sum_{j=-\infty}^{k-2} 2^{(k-j)\left(\alpha_{+}-n+\frac{n}{p_{-}}+\frac{n-1}{s}\right)}\left\|2^{j \alpha(\cdot)} f \chi_{j}\right\|_{L^{p(\cdot)}\left(\mathbb{R}^{n}\right)} .
\end{aligned}
$$


Noting that $\alpha_{+}-n+\frac{n}{p_{-}}+\frac{n-1}{s}<0$, we apply Lemma 2.4 and obtain

$$
\begin{aligned}
& \left\{\sum_{k=-\infty}^{-1}\left\|2^{k \alpha(0)} T_{\Omega, \beta}\left(f \chi_{B_{k-2}}\right) \chi_{k}\right\|_{L^{p^{*}(\cdot)}\left(\mathbb{R}^{n}\right)}^{q(0)}\right\}^{\frac{1}{q(0)}} \\
\leq & C\left\{\sum_{k=-\infty}^{-1}\left(\sum_{j=-\infty}^{k-2} 2^{(k-j)\left(\alpha_{+}-n+\frac{n}{p_{-}}+\frac{n-1}{s}\right)}\left\|2^{j \alpha(\cdot)} f \chi_{j}\right\|_{L^{p(\cdot)}\left(\mathbb{R}^{n}\right)}\right)^{q(0)}\right\}^{\frac{1}{q(0)}} \\
\leq & C\left\{\sum_{k=-\infty}^{-1}\left\|2^{k \alpha(0)} f \chi_{k}\right\|_{L^{p(\cdot)}\left(\mathbb{R}^{n}\right)}^{q(0)}\right\}^{\frac{1}{q(0)}} \\
\leq & C\|f\|_{\dot{K}_{p(\cdot), q(\cdot)}^{\alpha(\cdot)}\left(\mathbb{R}^{n}\right)^{\cdot}}
\end{aligned}
$$

To estimate $2^{k \alpha_{\infty}} T_{\Omega, \beta}\left(f \chi_{B_{k-2}}\right)$ in $\ell_{>}^{q_{\infty}}$-norm, we have the same estimate (3.4), with $2^{k \alpha_{\infty}}$ in place of $2^{k \alpha(0)}$. We write

$$
\begin{aligned}
& \quad\left\|2^{k \alpha_{\infty}} T_{\Omega, \beta}\left(f \chi_{B_{k-2}}\right) \chi_{k}\right\|_{L^{p^{*}(\cdot)}\left(\mathbb{R}^{n}\right)} \\
& \leq C \sum_{j=-\infty}^{k-2} 2^{(k-j)\left(\alpha_{+}-n+\frac{n}{p_{-}}+\frac{n-1}{s}\right)}\left\|2^{j \alpha(\cdot)} f \chi_{j}\right\|_{L^{p(\cdot)}\left(\mathbb{R}^{n}\right)} \\
& =C \sum_{j=-\infty}^{0} 2^{(k-j)\left(\alpha_{+}-n+\frac{n}{p_{-}}+\frac{n-1}{s}\right)}\left\|2^{j \alpha(\cdot)} f \chi_{j}\right\|_{L^{p(\cdot)}\left(\mathbb{R}^{n}\right)} \\
& \quad+C \sum_{j=1}^{k-2} 2^{(k-j)\left(\alpha_{+}-n+\frac{n}{p_{-}}+\frac{n-1}{s}\right)}\left\|2^{j \alpha(\cdot)} f \chi_{j}\right\|_{L^{p(\cdot)}\left(\mathbb{R}^{n}\right)},
\end{aligned}
$$

for any $k \geq 0$ (we put $\sum_{j=1}^{k-2} \cdots=0$ if $k=0,1,2$ ). Once again by Lemma 2.4 , we get

$$
\begin{aligned}
& \left\{\sum_{k=0}^{\infty}\left\|2^{k \alpha_{\infty}} T_{\Omega, \beta}\left(f \chi_{B_{k-2}}\right) \chi_{k}\right\|_{L^{p^{*}(\cdot)}\left(\mathbb{R}^{n}\right)}^{q_{\infty}}\right\}^{\frac{1}{q_{\infty}}} \\
\leq C & \left\{\sum_{k=0}^{\infty}\left(\sum_{j=-\infty}^{0} 2^{(k-j)\left(\alpha_{+}-n+\frac{n}{p_{-}}+\frac{n-1}{s}\right)}\left\|2^{j \alpha(\cdot)} f \chi_{j}\right\|_{L^{p(\cdot)}\left(\mathbb{R}^{n}\right)}\right)^{q_{\infty}}\right\}^{\frac{1}{q_{\infty}}} \\
& +C\left\{\sum_{k=0}^{\infty}\left(\sum_{j=1}^{k-2} 2^{(k-j)\left(\alpha_{+}-n+\frac{n}{p_{-}}+\frac{n-1}{s}\right)}\left\|2^{j \alpha(\cdot)} f \chi_{j}\right\|_{L^{p(\cdot)}\left(\mathbb{R}^{n}\right)}\right)^{q_{\infty}}\right\}^{\frac{1}{q_{\infty}}} \\
\leq C & \left\{\sum_{k=0}^{\infty}\left(2^{k\left(\alpha_{+}-n+\frac{n}{p_{-}}+\frac{n-1}{s}\right)} \sum_{j=-\infty}^{0} 2^{-j\left(\alpha_{+}-n+\frac{n}{p_{-}}+\frac{n-1}{s}\right)} \sup _{j \leq 0} 2^{j \alpha(0)}\left\|f \chi_{j}\right\|_{L^{p(\cdot)}\left(\mathbb{R}^{n}\right)}\right)^{q_{\infty}}\right\}^{\frac{1}{q_{\infty}}} \\
& +C\left\{\sum_{k=0}^{\infty}\left(\sum_{j=1}^{k-2} 2^{(k-j)\left(\alpha_{+}-n+\frac{n}{p_{-}}+\frac{n-1}{s}\right)}\left\|2^{j \alpha(\cdot)} f \chi_{j}\right\|_{L^{p(\cdot)}\left(\mathbb{R}^{n}\right)}\right)^{q_{\infty}}\right\}^{\frac{1}{q_{\infty}}}
\end{aligned}
$$




$$
\leq C\|f\|_{\dot{K}_{p(\cdot), q(\cdot)}^{\alpha(\cdot)}\left(\mathbb{R}^{n}\right)^{\cdot}}
$$

For $T_{\Omega, \beta}\left(f \chi_{\widetilde{R_{k}}}\right)$, using the $\left(L^{p(\cdot)}, L^{p^{*}(\cdot)}\right)$-boundedness of $T_{\Omega, \beta}$, we have

$$
\begin{aligned}
& \left\|T_{\Omega, \beta}\left(f \chi_{\widetilde{R_{k}}}\right)\right\|_{\dot{K}_{p^{*}(\cdot), q(\cdot)}^{\alpha(\cdot)}\left(\mathbb{R}^{n}\right)} \\
& \approx\left\|\left\{T_{\Omega, \beta}\left(2^{k \alpha(0)} f \chi_{\widetilde{R_{k}}}\right)\right\}\right\|_{\ell_{<}^{q(0)}\left(L^{p^{*}(\cdot)}\right)}+\left\|\left\{T_{\Omega, \beta}\left(2^{k \alpha_{\infty}} f \chi_{\widetilde{R_{k}}}\right)\right\}\right\|_{\ell_{>}^{q^{\infty}}\left(L^{p^{*}(\cdot)}\right)} \\
& \leq C\left(\left\|\left\{2^{k \alpha(0)} f \chi_{\widetilde{R_{k}}}\right\}\right\|_{\ell_{<}^{q(0)}\left(L^{p(\cdot)}\right)}+\left\|\left\{2^{k \alpha_{\infty}} f \chi_{\widetilde{R_{k}}}\right\}\right\|_{\ell_{>}^{q \infty}\left(L^{p(\cdot)}\right)}\right) \\
& \leq C\|f\|_{\dot{K}_{p(\cdot), q(\cdot)}^{\alpha(\cdot)}\left(\mathbb{R}^{n}\right)} \text {. }
\end{aligned}
$$

We proceed now to estimate $T_{\Omega, \beta}\left(f \chi_{\mathbb{R}^{n} \backslash B_{k+2}}\right)$. Given $x \in R_{k}, k<0$, we write

$$
\begin{aligned}
& 2^{k \alpha(0)}\left|T_{\Omega, \beta}\left(f \chi_{\mathbb{R}^{n} \backslash B_{k+2}}\right)(x)\right| \\
\leq & C 2^{k \alpha(0)} \int_{\mathbb{R}^{n} \backslash B_{k+2}} \frac{|\Omega(x-y)|}{|x-y|^{n-\beta}}|f(y)| d y \\
= & C 2^{k \alpha(0)} \sum_{j=k+3}^{\infty} \int_{R_{j}} \frac{|\Omega(x-y)|}{|x-y|^{n-\beta}}|f(y)| d y .
\end{aligned}
$$

Noting that

$$
|x-y|>2^{j-1}-2^{k}>2^{j-3}
$$

for $x \in R_{k}$ and $y \in R_{j}$, by Lemma 2.2, we have

$$
2^{k \alpha(0)}\left|T_{\Omega, \beta}\left(f \chi_{\mathbb{R}^{n} \backslash B_{k+2}}\right)(x)\right| \leq C \sum_{j=k+3}^{\infty} 2^{(k-j) \alpha_{-}-j(n-\beta)} \int_{R_{j}} 2^{j \alpha(y)}|\Omega(x-y)||f(y)| d y .
$$

An application of Hölder's inequality (2.2) gives

$$
\begin{aligned}
& \left\|2^{k \alpha(0)} T_{\Omega, \beta}\left(f \chi_{\mathbb{R}^{n} \backslash B_{k+2}}\right) \chi_{k}\right\|_{L^{p^{*}(\cdot)}\left(\mathbb{R}^{n}\right)} \\
\leq & C \sum_{j=k+3}^{\infty} 2^{(k-j) \alpha_{-}-j(n-\beta)}\left\|2^{j \alpha(\cdot)} f \chi_{j}\right\|_{L^{p(\cdot)}\left(\mathbb{R}^{n}\right)}\left\|\Omega(x-\cdot) \chi_{j}\right\|_{L^{p^{\prime}(\cdot)\left(\mathbb{R}^{n}\right)}}\left\|\chi_{k}\right\|_{L^{p^{*}(\cdot)}\left(\mathbb{R}^{n}\right)} .
\end{aligned}
$$

Similarly to (3.3), we have the estimate

$$
\begin{aligned}
& \left\|\Omega(x-\cdot) \chi_{j}\right\|_{L^{p^{\prime}(\cdot)}\left(\mathbb{R}^{n}\right)} \\
\leq & C\left\|\chi_{j}\right\|_{L^{p^{\prime}(\cdot)\left(\mathbb{R}^{n}\right)}}\left|B_{j}\right|^{-1 / s}\left(\int_{0}^{j^{j+1}} \int_{S^{n-1}}\left|\Omega\left(y^{\prime}\right)\right|^{s} d \sigma\left(y^{\prime}\right) \rho^{n-1} d \rho\right)^{\frac{1}{s}} \\
\leq & C\left\|\chi_{j}\right\|_{L^{p^{\prime}(\cdot)\left(\mathbb{R}^{n}\right)}} .
\end{aligned}
$$


Noting that

$$
\left|R_{j}\right|^{-\frac{1}{p\left(x_{j}\right)}}\left|R_{k}\right|^{\frac{1}{p\left(x_{k}\right)}} \leq \mathrm{C} 2^{(k-j) \frac{n}{p_{+}}}
$$

for $x_{j} \in R_{j}, x_{k} \in R_{k}$ and $j \geq k+3$ (see [16, Lemma 3.2]), from (3.6), (3.7) and Lemma 2.3, we get

$$
\begin{aligned}
& \left\|2^{k \alpha(0)} T_{\Omega, \beta}\left(f \chi_{\mathbb{R}^{n} \backslash B_{k+2}}\right) \chi_{k}\right\|_{L^{p^{*}(\cdot)}\left(\mathbb{R}^{n}\right)} \\
\leq & C \sum_{j=k+3}^{\infty} 2^{(k-j) \alpha_{-}-j(n-\beta)}\left\|2^{j \alpha(\cdot)} f \chi_{j}\right\|_{L^{p(\cdot)}\left(\mathbb{R}^{n}\right)}\left\|\chi_{j}\right\|_{L^{p^{\prime}(\cdot)\left(\mathbb{R}^{n}\right)}}\left\|\chi_{k}\right\|_{L^{p^{*}(\cdot)}\left(\mathbb{R}^{n}\right)} \\
\leq & C \sum_{j=k+3}^{\infty} 2^{(k-j)\left(\alpha_{-}-\beta\right)}\left\|2^{j \alpha(\cdot)} f \chi_{j}\right\|_{L^{p(\cdot)}\left(\mathbb{R}^{n}\right)}\left|R_{j}\right|^{-\frac{1}{p\left(x_{j}\right)}}\left|R_{k}\right|^{\frac{1}{p\left(x_{k}\right)}} \\
\leq & C \sum_{j=k+3}^{\infty} 2^{(k-j)\left(\alpha_{-}+\frac{n}{p_{-}}-\beta\right)}\left\|2^{j \alpha(\cdot)} f \chi_{j}\right\|_{L^{p(\cdot)}\left(\mathbb{R}^{n}\right)^{\prime}}
\end{aligned}
$$

Observing that

$$
\alpha_{-}+\frac{n}{p_{-}}-\beta>0 \quad \text { and } \quad 2^{j \alpha(y)} \approx 2^{j \alpha_{\infty}} \text {, }
$$

for any $y \in R_{j}, j \geq 0$. Then Lemma 2.4 implies that

$$
\begin{aligned}
& \quad\left\{\sum_{k=-\infty}^{-1}\left\|2^{k \alpha(0)} T_{\Omega, \beta}\left(f \chi_{\mathbb{R}^{n} \backslash B_{k+2}}\right) \chi_{k}\right\|_{L^{p^{*}(\cdot)}\left(\mathbb{R}^{n}\right)}^{q(0)}\right\}^{\frac{1}{q(0)}} \\
& \leq C\left\{\sum_{k=-\infty}^{-1}\left(\sum_{j=k+3}^{-1} 2^{(k-j)\left(\alpha_{-}+\frac{n}{p_{+}}-\beta\right)}\left\|2^{j \alpha(\cdot)} f \chi_{j}\right\|_{L^{p(\cdot)}\left(\mathbb{R}^{n}\right)}\right)^{q(0)}\right\}^{\frac{1}{q(0)}} \\
& +C\left\{\sum_{k=-\infty}^{-1}\left(\sum_{j=0}^{\infty} 2^{(k-j)\left(\alpha_{-}+\frac{n}{p_{+}}-\beta\right)}\left\|2^{j \alpha(\cdot)} f \chi_{j}\right\|_{L^{p(\cdot)}\left(\mathbb{R}^{n}\right)}\right)^{q(0)}\right\}^{\frac{1}{q(0)}} \\
& \leq C\left\{\sum_{k=-\infty}^{-1}\left\|2^{k \alpha(0)} f \chi_{k}\right\|_{L^{p(\cdot)}\left(\mathbb{R}^{n}\right)}^{q(0)}\right\}^{\frac{1}{q(0)}} \\
& +C\left\{\sum_{k=-\infty}^{-1}\left(2^{k\left(\alpha_{-}+\frac{n}{p_{+}}-\beta\right)} \sum_{j=0}^{\infty} 2^{-j\left(\alpha_{-}+\frac{n}{p_{+}}-\beta\right)} \sup _{j \geq 0} 2^{j \alpha_{\infty}}\left\|f \chi_{j}\right\|_{L^{p(\cdot)}\left(\mathbb{R}^{n}\right)}\right)^{q(0)}\right\}^{\frac{1}{q(0)}} \\
& \leq C\|f\|_{\dot{K}_{p(\cdot), q(\cdot)}^{\alpha(\cdot)}\left(\mathbb{R}^{n}\right)}
\end{aligned}
$$

To estimate $2^{k \alpha_{\infty}} T_{\Omega, \beta}\left(f \chi_{\mathbb{R}^{n} \backslash B_{k+2}}\right)$ in $\ell_{>}^{q_{\infty}}$-norm, we have the same estimate (3.4), with $2^{k \alpha_{\infty}}$ in place of $2^{k \alpha(0)}$. We write

$$
\left\|2^{k \alpha_{\infty}} T_{\Omega, \beta}\left(f \chi_{\mathbb{R}^{n} \backslash B_{k+2}}\right) \chi_{k}\right\|_{L^{p^{*}(\cdot)}\left(\mathbb{R}^{n}\right)} \leq C \sum_{j=k+3}^{\infty} 2^{(k-j)\left(\alpha_{-}+\frac{n}{p_{-}}-\beta\right)}\left\|2^{j \alpha(\cdot)} f \chi_{j}\right\|_{L^{p(\cdot)}\left(\mathbb{R}^{n}\right)} .
$$


This together with Lemma 2.4 yields the desired inequality

$$
\begin{aligned}
& \left\{\sum_{k=0}^{\infty}\left\|2^{k \alpha_{\infty}} T_{\Omega, \beta}\left(f \chi_{\mathbb{R}^{n} \backslash B_{k+2}}\right) \chi_{k}\right\|_{L^{p^{*}(\cdot)}\left(\mathbb{R}^{n}\right)}^{q_{\infty}}\right\}^{\frac{1}{q_{\infty}}} \\
\leq & C\left\{\sum_{k=0}^{\infty}\left(\sum_{j=k+3}^{\infty} 2^{(k-j)\left(\alpha_{-}+\frac{n}{p_{+}}-\beta\right)}\left\|2^{j \alpha(\cdot)} f \chi_{j}\right\|_{L^{p(\cdot)}\left(\mathbb{R}^{n}\right)}\right)^{q_{\infty}}\right\}^{\frac{1}{q_{\infty}}} \\
\leq & C\|f\|_{\dot{K}_{p(\cdot), q(\cdot)}^{\alpha(\cdot)}\left(\mathbb{R}^{n}\right)^{\prime}}
\end{aligned}
$$

The proof of Theorem 3.2 is completed.

\section{Acknowledgements}

Supported by Natural Science Foundation of Education Committee of Anhui Province (No. KJ2019A1196), Anhui Provincial Natural Science Foundation (No. 1908085MA19) and Pre-research Project of the NNSF of China (No. 2019yyzr14).

\section{References}

[1] A. Almeida, and D. Drihem, Maximal, potential and singular type operators on Herz spaces with variable exponents, J. Math. Anal. Appl., 394 (2012), 781-795.

[2] A. Almeida and P. Höstö, Besov spaces with variable smoothness and integrability, J. Funct. Anal., 258 (2010), 1628-1655.

[3] Y. Chen, S. Levine and R. Rao, Variable exponent, linear growth functionals in image restoration, SIAM J. Appl. Math., 66 (2006), 1383-1406.

[4] D. Cruz-Uribe and A. Fiorenza, Variable Lebesgue Spaces: Foundations and Harmonic Analysis, Applied and Numerical Harmonic Analysis, Birkhöuser, Basel, 2013.

[5] L. Diening, P. Harjulehto, P. Höstö and M. Rúžička, Lebesgue and Sobolev Spaces with Variable Exponents, volume 2017 of Lecture Notes in Mathematics, Springer, Heidelberg, 2011.

[6] D. Drihem and F. Seghiri, Notes on the Herz-type Hardy spaces of variable smoothness and integrability, Math. Inequal. Appl., 19 (2016), 145-165.

[7] P. Harjulehto and P. Höstö, V. LêÚ and M. Nuortio, Overview of differential equations with non-standard growth, Nonlinear Anal., 72 (2010), 4551-4574.

[8] K.-P. Ho, The fractional integral operators on Morrey spaces with variable exponent on unbounded domains, Math. Inequal. Appl., 16 (2013), 363-373.

[9] K.-P. Ho, Weak type estimates of the fractional integral operators on Morrey spaces with variable exponents, Acta Appl. Math., 159 (2019), 1-10.

[10] G. Hu, S. Lu and D. Yang, Boundedness of rough singular integral operators on homogeneous Herz spaces, J. Austral. Math. Soc., 66 (1999), 201-223.

[11] M. Izuki, Commutators of fractional integrals on Lebesgue and Herz spaces with variable exponent, Rend. Circ. Mat. Palermo., 59 (2010), 461-472.

[12] O. Kováčik and J. Rákosník, On spaces $L^{p(x)}$ and $W^{k, p(x)}$, Czechoslovak Math. J., 41 (1991), 592-618. 
[13] S. $\mathrm{Lu}$ and $\mathrm{L} . \mathrm{Xu}$, Boundedness of rough singular intergral operators on the homogeneous Morrey-Herz spaces, Hokkaido Math. J., 34 (2005), 299-314.

[14] S. Lu, D. Yang and G. Hu, Herz Type Spaces and Their Applications, Science Press, 2008.

[15] S. Lu, D. Yang and Z. Zhou, Sublinear operators with rough kernel on generalized Morrey spaces, Hokkaido Math. J., 27 (1998), 219-232.

[16] Y. Lu and Y. Zhu, Boundedness of multilinear Calderón-Zygmund singular operators on Morrey-Herz spaces with variable exponents, Acta Math. Sin., 30 (2014), 1180-1194.

[17] E. Nakai and Y. Sawano, Hardy spaces with variable exponents and generalized Campanato spaces, J. Funct. Anal., 262 (2012), 3665-3748.

[18] M. Ragusa, Homogeneous Herz spaces and regularity results, Nonlinear Anal., 71 (2009), 1909-1914.

[19] M. Rúžička, Electrorheological Fluids: Modeling and Mathematical Theory, Springer-Verlag, Berlin, 2000.

[20] F. Soria and G. Weiss, A remark on singular integrals and power weights, Indiana Univ. Math. J., 43 (1994), 187-204. 